\title{
A Comprehensive Review on Data Dissemination Techniques in VANET
}

\author{
Harikrishna ${ }^{1}$, Kuldeep Kumar $^{2}$ and Sandeep Kumar Arora ${ }^{3}$ \\ Department of Electronics and Communication Engineering, \\ Lovely Professional University, Jalandhar - 144411, Punjab, India \\ hrkrishna116@gmail.com ${ }^{1}$,kuldeep.etah@yahoo.com ${ }^{2}$,sandeep.16930@lpu.co.in ${ }^{3}$
}

\begin{abstract}
Vehicular Ad-hoc Network(VANET) is growing field of Intelligent transportation system having the feature of wireless networks. In VANET, communication takes place among Vehicle to Vehicle(V2V) or Vehicle to road-side Infrastructure(V2I). It has the capability to handle traffic condition and reduce road accidents. VANET helps in the dissemination of road conditions and traffic information on moving vehicles. Data dissemination is used for transferring the messages as well as for improving the driving quality in term of distance, time, and safety from source to destination vehicles. In this paper, we discuss the various data dissemination technique and routing protocol used in VANET.
\end{abstract}

Keywords: Data dissemination, accidents, security, safety, routing protocol

\section{Introduction}

Vehicular Ad-hoc Network (VANET) mainly focus on improving the road safety and travel convenience. Without requiring a centralized administration or a fixed infrastructure, VANET provides a self-organizing network. In VANET, vehicle communicates with each other and exchange information about travel situation and road conditions. In the near future, VANET is expecting the more increasing number of the vehicle equipped with communication capabilities. VANET are basically based on short range communication [1]. Recently allocation of $75 \mathrm{MHz}$ in the $5.9 \mathrm{GHz}$ licensed Dedicated Short Range Communication (DSRC) was made by Federal Communications Commission (FCC). VANET is classifieds into two applications: Safety applications and non-safety applications. In safety application, we deal with work zone warning and collision warning [2]. In VANET vehicle send their request to other vehicles get back reply within a fraction of second hence communication is multi-hop. Data dissemination is the brainchild of VANET communication. The purpose of data dissemination technique is to utilize the network resource maximum possible and fulfill all user requirement. In VANET two type of communication is possible, first in which delay is tolerable like commercial advertisements, parking condition at the parking place, estimated arrival time of the bus at a stop, remaining stock status at the commercial stores, schedule of the meeting etc. Second in which delay is not tolerable like a road accident, traffic condition and safety messages [3]. VANET is self-organized, distributed and potentially highly mobile networks of vehicles communicating via wireless media. In VANET every vehicle act as both router as well as host node due to frequent disconnection and high mobility. As there is a frequent disconnection in VANET, we cannot use routing protocol used in Mobile Ad hoc Network(MANET)because, in MANET, there is an implicit assumption of network connectivity.

Routing is used to find out an optimal path between source and destination and message is send through this path to the destination. The main problem in VANET is an exchange of information in a scalable fashion which needs to be solved. The solution to 
this problem is Data Dissemination Protocols. Application of data dissemination protocols have different scenarios: first is highway while others are used in urban areas and some of them can be used in both scenarios. There are a lot of intersection points and large buildings in the urban areas which can block the communication among vehicle near to the building. To avoid this situation, we send messages to vehicle or Road Side Unit (RSU) so that information should not be lost and can reach to the vehicle. Data dissemination divided into the following category as shown in Figure 1.

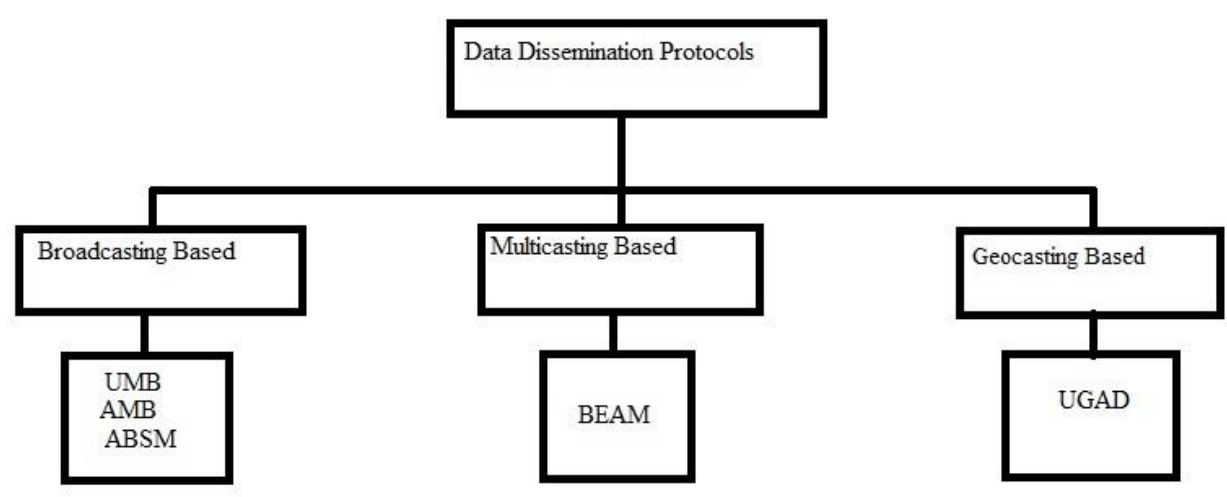

Figure 1. Data Dissemination Protocol

\section{Challenges in Data Dissemination}

Data dissemination is a technique of distributing information or data over distributed networks. Thus, data dissemination is used to improve the efficiency and accuracy of traffic management in VANET. It also helps in quality driving. Though data dissemination process appears to be very simple but in actual, it is difficult for vehicles to communication takes place among themselves due to a large number of vehicles on road. It is very challenging work for vehicles to send information over the network. Problem related to data dissemination are.

\subsection{High Mobility and Frequent Disconnections}

The high mobility and frequently disconnected topologies are the main challenges in VANET for different regions in the city. In daytime during peak hour, the traffic density is very high. For suburban area traffic density is low during night. For all the recipients distributed across the city, there is no simple solution for everyone during the dissemination of data [4].

\subsection{Data Transmission in Presence of Disconnection}

In VANET, how to disseminate data with less delay to the new vehicle which enters in the network before get disconnected is another challenging task. Disconnection is less when target vehicle comes closer to RSU and placed in the dense area. But the biggest problem occurs when large no of different vehicle demanding for same information at the same time within network range. In this situation, the allocation of bandwidth and sharing of wireless medium is the challenging task. When Vehicles pass through RSU within single hope, the data can be transmitted to such vehicle with high throughput. Hence, we can extend most desirable connection time between the vehicle and RSU to distribute more data. 


\subsection{Data Distribution over the Mesh Nodes}

To disseminate data to the vehicles effectively, roadside unit forms mesh infrastructure as shown in Figure 2. Hence it is difficult how to distribute the data.

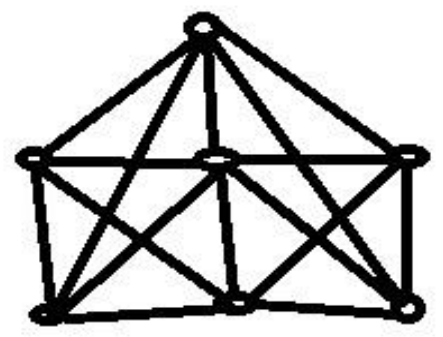

Figure 2. Mesh Network

\section{Data Dissemination Technique}

Data dissemination is a technique of spreading of information or data over distributed network wirelessly. The aim of data dissemination is to focus on the efficient usage of network resources and to provide the data service according to user wish. Types of data dissemination are as follows:

3.1 Vehicle to infrastructure or RSU dissemination (V2I/I2V Dissemination)

3.2 Vehicle to vehicle (V2V) dissemination

3.3 Opportunistic Dissemination

3.4 Peer-to-peer Dissemination

3.5 Cluster-Based Dissemination

\subsection{Vehicle to Infrastructure (V2I/I2V) Dissemination}

Vehicular to infrastructure dissemination are two type: Push-based and Pull based. Concepts of data pouring and buffering are used in Push based data dissemination. In data pouring technique usually, a road with the highly mobile vehicle is selected and it is the responsibility of data center to broadcast the information to the vehicles running on the same road as well as on crossing roads. The work of data center is the collection of data from outside and deliver it to the vehicles within its wireless interface. Buffer are used to storing the data and it collects the data from buffer installed at intersection points, these data are then transmitted to the moving vehicle on the road. Hence, in push-based data dissemination, data is efficiently transmitted from the RSUs (Road Side Units) or moving vehicles to another vehicle but we use pull-based data dissemination technique only when the vehicle needs any information from RSU or vehicle. Generally, this scheme used for query purpose [4].

\subsection{V2V Dissemination}

In V2V data dissemination relaying and flooding technique are used. Generally, in flooding technique, data is broadcasted to all nodes those are participating in data dissemination process. In this type of data communication technique, one-for-all communication takes place. In the case of the relay data dissemination relay node is selected then it is used to forwarding the data to next relay hop and so on. mechanism of technique issued for congested networks [5].

\subsection{Opportunistic Dissemination}

In this case of opportunistic dissemination technique, intermediate nodes are used for message storage and each message is forwarded to every next node until the destination is 
reached [5].

\subsection{Peer to Peer Dissemination}

In Peer to Peer dissemination technique, the source node is used like a storage device to stores the data and forward this data to the network only when it is demanded by another vehicle [5].

\subsection{Cluster-Based Dissemination}

Cluster heads are elected and with the help of cluster member nodes communication is possible or more node collects data in its cluster and forward them to the next cluster [5].

Table 1. Comparison of Various Data Dissemination Technique

\begin{tabular}{|l|l|l|l|}
\hline $\begin{array}{l}\text { Dissemination } \\
\text { Type }\end{array}$ & $\begin{array}{l}\text { Dissemination } \\
\text { Approach }\end{array}$ & \multicolumn{1}{|c|}{ Pros } & \multicolumn{1}{|c|}{ Cons } \\
\hline V2I/I2V & $\begin{array}{l}\text { Push based } \\
\text { technique } \\
\text { technique }\end{array}$ & $\begin{array}{l}\text { It is suitable for } \\
\text { popular data }\end{array}$ & $\begin{array}{l}\text { It is not suitable for } \\
\text { non-popular data }\end{array}$ \\
\hline V2V & Flooding & $\begin{array}{l}\text { It is suitable for } \\
\text { on popular data, } \\
\text { user-specific data. }\end{array}$ & $\begin{array}{l}\text { In this cross traffic } \\
\text { Incurs and } \\
\text { interferences and } \\
\text { collisions }\end{array}$ \\
\hline Relaying & $\begin{array}{l}\text { Data distribution } \\
\text { is done quickly } \\
\text { and reliability }\end{array}$ & $\begin{array}{l}\text { Not suitable for } \\
\text { dense networks }\end{array}$ \\
\hline Opportunistic & Store and forward & $\begin{array}{l}\text { It works well in } \\
\text { anse networks } \\
\text { and in congested } \\
\text { networks }\end{array}$ & $\begin{array}{l}\text { It is used for } \\
\text { selecting next best } \\
\text { hop and reliability is } \\
\text { difficult }\end{array}$ \\
\hline P2P & $\begin{array}{l}\text { Routes are built } \\
\text { Store and forward }\end{array}$ & $\begin{array}{l}\text { It has data centric } \\
\text { architecture in which } \\
\text { applications are not } \\
\text { concerned } \\
\text { transportation of data } \\
\text { to the right place }\end{array}$ \\
\hline $\begin{array}{l}\text { Cluster-based } \\
\text { dissemination }\end{array}$ & $\begin{array}{l}\text { Clusters are } \\
\text { generated } \\
\text { applications }\end{array}$ & $\begin{array}{l}\text { It provides high } \\
\text { delivery ratio and } \\
\text { delay is less }\end{array}$ & $\begin{array}{l}\text { Messages are not } \\
\text { sent in the network } \\
\text { allowed to broadcast } \\
\text { the messages }\end{array}$ \\
\hline
\end{tabular}

\section{Broadcasting Based Data Dissemination Protocols in VANETs}

The previous few years, so many broadcasting protocol for VANET are reportable within the literature. They will be typically classified into two main classes as per spreading of data packets within the network. [6]

\subsection{Single Hop Broadcasting Protocols}

In Single hop broadcasting Protocols, vehicles sporadically disseminate a large number of the data as info to the opposite vehicle within the networks. Broadcasting intervals and knowledge square measure the two selections that require being thought about whereas planning the printed protocols for VANETs. To stay the foremost up to date info while 
not redundancies, printed intervals should be set fitly. It must not be too short and too long. Aside from after this, relevant and vital info ought to solely be designated to broadcasting. In the Single hop broadcasting, protocols will be additional divide into planned approach parts.

\subsubsection{Fixed Interval Based Single Hop Broadcasting Protocols}

In case of fixed interval based broadcasting protocol, single hop broadcasting protocols focus on aggregation of information and its choice. Traffic Info [7] is associated with sample of mounted broadcasting interval protocols within which each vehicle supplied with a digital road map and Global Positioning System (GPS) and sporadically broadcast the traffic information keep in its specific style. The reportable traffic information travel time segment throughout broadcast. Every vehicle stores its period of time and times taken by alternative vehicle throughout the travel. Traffic Info usages information measured with efficiency and broadcasts solely. The connection of the data is decided by a ranking rule, that relies on the present locations of the vehicles and current time.

Traffic View is an alternative type of single hop fastened interval broadcast [6] theme design for sanction native interchange of traffic info amongst vehicle. Position and Speed are two info sorts that area unit changed amongst the vehicle. During this theme, once a vehicle receives broadcasting packets, it initially stores the info in its databases and the info is then rebroadcasted within the subsequent next broadcasting cycles. However, rather than broadcast all hold on records from info, solely one records are broadcasting when aggregate the multiples record. Cost based and the Ratio based area unit are two algorithms for aggregation. Within the Cost based rule, the price will be thought to be the loss of accuracy incurred from combining the record. Whereas in the ratio based rule, roads are split into the tiny region, and an aggregations quantitative relations are appointed to every region in step with the level of accuracy needed for that region and also the importance of the dense. In this, both the simulation shows that though the costbased rule produces higher accuracy, the ratio based rule offers additional flexible.

\subsubsection{Adaptive Interval Based Single Hop Broadcasting Protocols}

In this type, adaptive broadcast interval single hop broadcasting protocol, associate degree adjustments of broadcasting intervals are additionally take into account. Collision Ratio Control Protocol (CRCP) [9] use adjuster broadcasting interval within every vehicle disseminate the traffic info sporadically. The traffics info during in this case is the speed road ID and location which is measured at each second. This protocol employs a mechanism for dynamically changed published interval and support a number of packets collision. Essentially, the protocols aim at keeping the collisions quantitative relation at target levels notwithstanding the vehicles density. Instinctively, a number of packets collision will increase a rise in networks thickness except for adjuster broadcasting interval mechanisms, three methods are: Random choice, Vicinity Priority Selection with Queries (VPSQ) and Vicinity Priority Selection (VPS) square measure planned for choosing the info to be disseminated.

In this, another type of protocol namely Segment Oriented Data Abstraction and Dissemination (SODA) protocols [13] conjointly use adaptation broadcasting interval within which road is divided into the segment of predefined lengths. All vehicles collect the info by sense the data himself and from the report of the alternative vehicle. All vehicles adaptive adjust its broadcasting interval scale back redundancy info receive from the alternative vehicle is characterize in two methods.
(i) Mollification
(ii) Provocation

A mollification event is outlined as an occasion that will increase the times till next broadcasted, whereas a provocation event is an occasion that reduces the time till next broadcasted. Once vehicles receive packets, it defines whether or not it's a mollification or 
provocation events by assignment a weight to the receive packets. A weight is calculating from the inconsistency between the receive information and people within the vehicle's information. The burden is going to be higher if the received info is original than the keep info supported the packets weights, nodes determine whether or not a mollification or provocation events has occurred by scrutiny with the threshold. The times for afterward beam is enlarged or faded counting on the burden.

\subsection{Multi-Hop Broadcasting Protocols}

In this multi-hop broadcasting protocols, flood is employed for packets propagations within all or networks. On the other hand, the pure flood is inefficient as a result of its lack quantifiable and their heap of packets collisions. Redundancy will increase because networks become thicker and waste channels information measure that successively reduces the network quantifiably. Additionally, packet collision is another vital downside as a result of multiples vehicle within the similar regions might transmit the packets an equivalent time. This is often referred to as broadcasting storm [6] downside. The multihop broadcast will be more divided into following three classes.

\subsubsection{Delay Based Multi-Hop Broadcasting Protocols}

Delay based multi-hop broadcast protocol theme, totally dissimilar waiting time before rebroadcasting the packets is allotted to every receive vehicles. Essentially, vehicles have a short waiting time get the best priority to broadcast the packets. Additionally, redundancy is avoided by the opposite vehicle to abort there waiting method once they recognize that packets have al-ready been rebroadcast. Whereas totally dissimilar delay area unit allotted to every vehicle in the delay based broadcast protocol, a unique broadcast chance is allotted every vehicle in very probabilistic based protocols.

In this type of Urban Multi-Hop Broadcast (UMB) protocols [4] could be a delay base mostly multi-hop broadcast protocols design to unravel printed storm, the unseen terminals, also the dependableness issues in multi-hop broadcast. UMB divided roads within the transmissions vary of transmitters into the lesser segment, and it offers the transmit priority to the vehicle that belongs to the furthest section. UMB use two kinds of packets forward.

(i) Intersection broadcast, and

(ii) Directional Broadcast

UMB as a result of is inefficient, next transmit vehicle needs to wait the long before having the ability to transmits clear to broadcast (CTB) packets. There can be as a result of the long black burst period is assign to following transmit vehicles as shown in Figure 3.

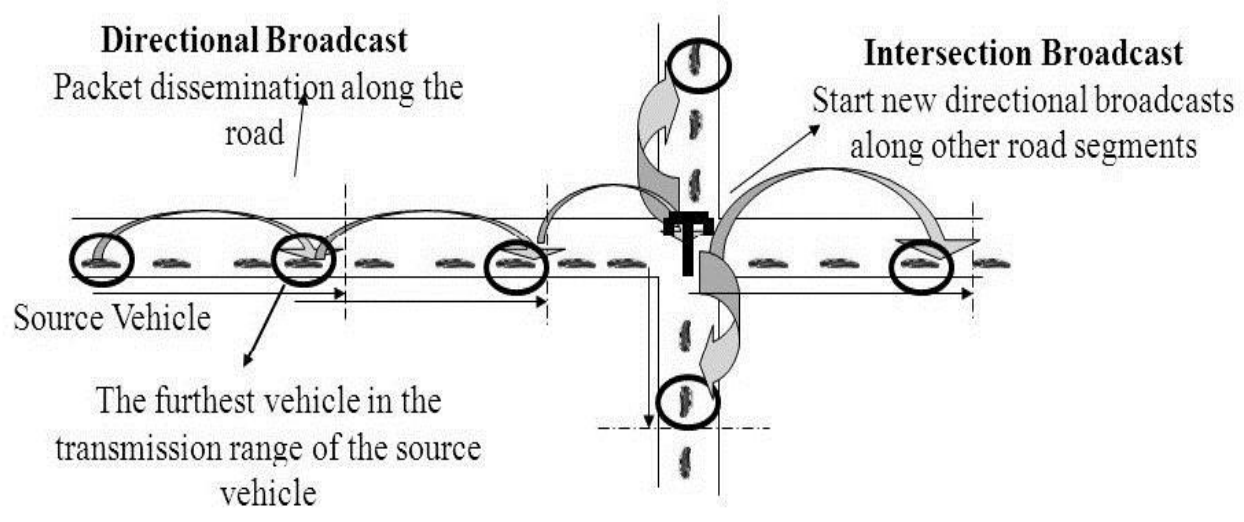

Figure 3. Urban Multihop Broadcast (UMB) Protocol 
In this another type of delay based multi-hop broadcasting protocol of Efficient Directionally Broadcasting (EDB) protocols [12] that work somewhat kind of like SB (Smart Broadcast) and (UMB) protocol. However, it doesn't use CTB and RTB (Request to Broadcast) management packet. EDB can jointly exploit employment of directional antenna. In specific, it's projected that every vehicle is supplied with two directionally antennas, for each with 30 (Thirty) degree beams breadth. Like UMB protocol, EDB conjointly uses two kinds of packets forward, specifically directionally broadcasted on the roads phase and directional broadcasting at the intersections.

Fastest Vehicles is one more Multi-Hop routing protocols. It used speeds data of every one vehicle for messages transfers and distances of the chosen vehicles from the last stop vehicles. On the premise of speeds of the vehicle and distances of the vehicle from the destinations, the time for every one vehicle inside the transmissions vary is calculated. The vehicles with the smallest amount time are chosen because of the subsequent or next hop for data disseminations.

\subsubsection{Probability Based Multi-Hop Broadcasting Protocol}

In probability based broadcast protocol approaches, every vehicle rebroadcast packets in keeping with the assign probabilistic. Since solely few vehicles can beam the packets, packet collisions and redundancy are reduced. The third class of multi-hop broadcasting is networks cryptography that has caught attentions within the fields of unintended wireless communication.

In Optimized Adaptive Probabilistic Broadcast (OAPB) protocols, [8] during which range of neighbor is that native vehicles density is additionally taken into considering whereas decisive the forward chance. Every One vehicle exchange Hello packet sporadically for picking associate degree applicable forwarding chance. specifically, when a vehicle receives a packet; it computes its own forward chance supported the subsequent equations.

$$
\tilde{\mathrm{O}}=(\mathrm{P} 1+\mathrm{P} 2+\mathrm{P} 3) / 3
$$

where $\mathrm{P} 1, \mathrm{P} 2$, and $\mathrm{P} 3$ area unit function of the amount of one hop neighbor, the amount of two hop neighbor, and a group of 2 hop neighbor that may solely be reached through a selected one hop neighbors.

\subsubsection{Networks Coding Based Multi-Hop Broadcasting Protocol}

Networks code writing could be new means of data disseminations which may be applied to a settled broadcasting approach, leading to vital reduction within the range of transmission networks and therefore yields highest out turn than the normal technique of transmissions.

CODE is a network coding based Multi-hop broadcast protocols [11] introduce. It extends the ideas and techniques planned in technique COPE to covers broadcast eventualities in wireless ad hoc network. It used timeserving listen, wherever all and each node snoop all packet overheard by it. additionally, every node sporadically broadcast the list of its one-hop neighbor. This enables all node to make an inventory of its two-hop neighbor, which is able additional be accustomed constructs some broadcast backbones. Furthermore, CODEB depends on time serving secret writing, during which secret writing opportunities to transmits code packet is decided. CODEB additionally realized timeserving secret writing for broadcasting is rather totally different from secret writing for unicasts. In broadcast, all the neighbor of the nodes should receive the packets wherever as in unicast, solely meant subsequent or next hop node receives a given packet. Thus, broadcast will increase the amount of complexness as all node that receives packets should be ready to rewrite.

DifCode is additionally a networks coding based multi-hop broadcast protocols. Its goals were cut back the quantity of transmission needed to flooding packet in wireless ad 
hoc networks almost like CODEB, DifCode conjointly chooses the future forward node to determine. However, DifCode is use variety formula supported multipoint relay (MPR). Multipoint relay [6] of nodes are that the list of its one-hop neighbor that cover its twohop zone. In DifCode, the node will encode and broadcast solely that packet that square measure receives from those nodes that choose it as their MPR.CODEB [13] and DifCode conjointly disagree by their expedient secret writing technique. In CODEB, all neighbor of a transmitters decryption the receive packet straightaway and hence limits secret writing opportunities. On the opposite hands, DifCode relaxes this constraint by permitting a node to buffer packet that isn't straightaway decodable. especially, all node can maintain a buffer for keep 3 differing kinds of packets:

(i) with success decode packet.

(ii) not straight away decodable packet, and

(iii) packet that require to be encoded and broadcast additional. Simulations result conjointly show that DifCode conjointly ends up in lowers redundancy rate than the probabilistic broadcast protocol.

\section{Multicasting Based Data Dissemination Protocols in VANET}

The aim of this protocol is to utilize information measure expeditiously throughout associate degree emergency state of affairs by minimizing the total variety of in networks message transaction. Bandwidth efficient Acknowledge based Multicasting Protocols (BEAM) is formed rather than existing broadcast than broadcast like acknowledgments broadcasting from static to extremely mobiles associate degree reconciling broadcast protocol. BEAM protocol will multicasting rather than broadcast i.e. rather than doing one to all or any communications, it is transmitting the message to solely this vehicle that wishes to receives that message. Road Side Units (RSUs) plays an important part during this protocol. [4]

\subsection{Overview of BEAM Protocol}

RSUs (Road Side Units) can begin the disseminations of the packet. Firstly, of all RSUs can broadcasting be part of packets to all the vehicle in their transmissions range. Once obtaining be part of the packet, vehicles that have the interest to speak with RSUs can reply back to explicit RSUs. Nowadays, RSUs can produce Multicasting cluster that contains all those vehicles that had replied to RSUs and everyone communications are done between RSUs and multicasting vehicle in multicasting us rather. Standing timer concerning by RSUs which resolve in all probability of second among that point all vehicle in multicast cluster got to tells about their standing (i.e. concerning yaw rate and speeds) to RSUs, the vehicle fails to try to this may not be a part of this cluster. Once obtaining standing reports if it is observing growth in speeds it means that one thing went wrongs and propagates emergency warning message (EWM) [10] to the vehicle within the cluster and obtain acknowledgments from them, if not get acknowledgments among the acknowledgement timer then transmit the messages to explicit vehicles in order that it will privacy to emergency state of affairs and take preventives action. As emergency message square measure multicasting, rather than broadcasting, therefore, this protocol utilizes information measure with efficiency throughout emergency things that it's called Bandwidth Efficient Acknowledgment based Multicasting Protocol (BEAM) as shown in Figure 4 . 


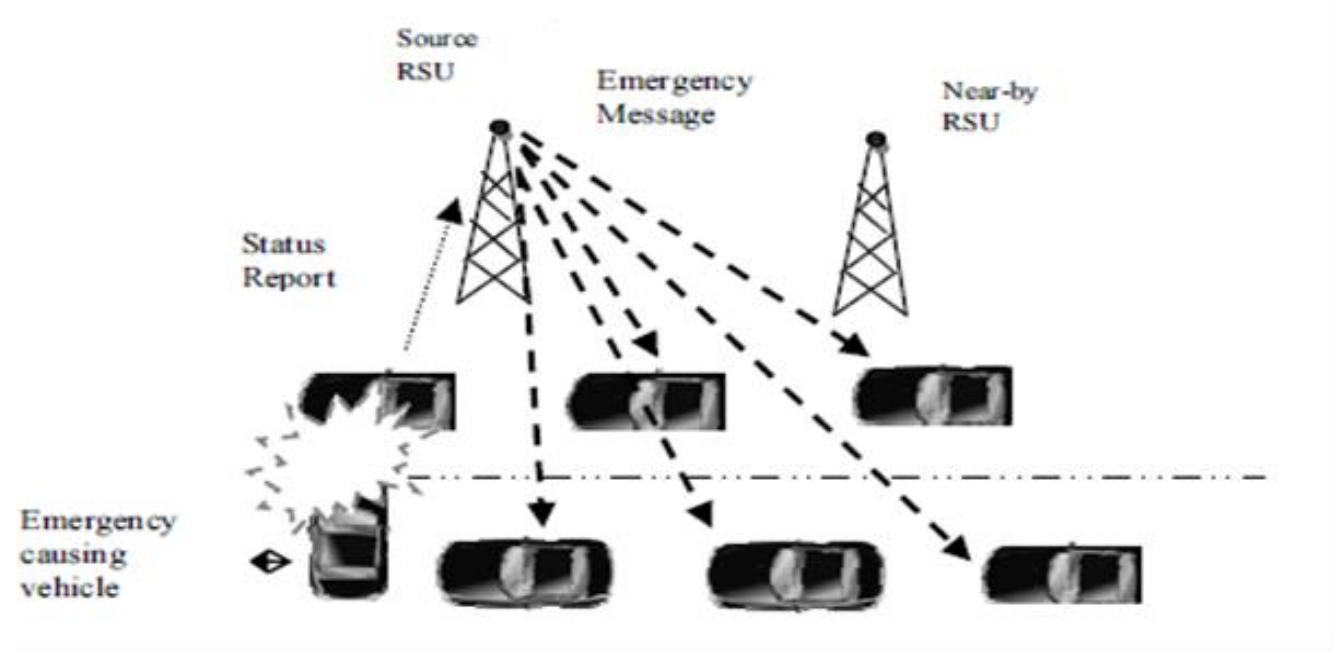

Figure 4. Illustrations of Emergency Events

\section{Adaptive Delay Based Geocast Protocols in Urban VANETs}

Instead of broadcast, geo cast protocols is employed during in this paper. Broadcast mean that sender nodes flood the packet to any or all node that are in its communications vary that ends up in broadcasting storm downside. So as to avoids this downside, broadcasting suppression ways are use that reis totally different in several eventualities and most of the present suppression ways don't add an urban environment. One exists delay based mostly suppression theme is customized during this paper in order that creating the protocols appropriate for the urban environment. Broadcasting based mostly geo cast allows economical knowledge disseminations to the vehicle inside the given geographical region. [14] Before this protocol, nobody has taken into consideration the urban area for suppression mechanisms. Once staring at deep within it come out that the majority of existing broadcasting suppression ways don't add urban areas as a result of in urban area there are many varieties of intersection and building offered and building will block direct communications of vehicle whether or not they are in transmissions vary of every alternative as shown in Figure 5.

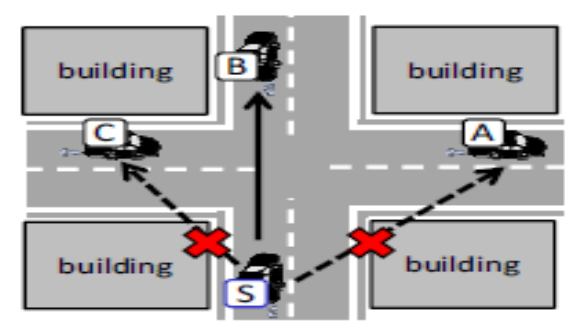

(a) Broadcast on road

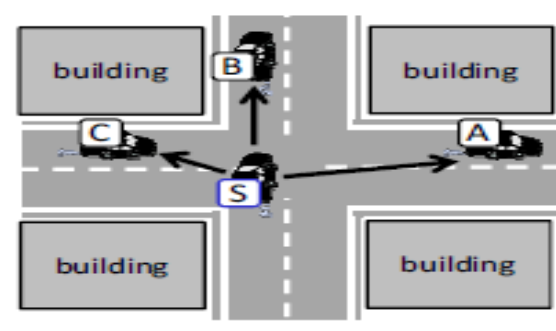

(b) Broadcast at intersection

Figure 5. Broadcast in Urban Environments

Nowadays this protocol name Urban Geocasting base on adaptive Delay (UGAD) says that as building square measure act as an obstacle for information disseminations thus there is got to transfers information to that vehicle that may be seen direct; no building or not the other obstacles is there. And a lot of variety of vehicle that may be seen direct by sources vehicles once another source vehicle reaches the intersection. [14] So, in keeping with this protocols chance for causation information at the intersection shouldn't be incomprehensible as shown in Figure 5(b). 
Table 2. Comparison of Data Dissemination Protocols

\begin{tabular}{|c|c|c|c|c|c|}
\hline $\begin{array}{l}\text { Existing Protocol } \\
\text { Name }\end{array}$ & $\begin{array}{l}\text { Mechanism } \\
\text { used }\end{array}$ & Pros & Cons & Highways & Urban \\
\hline $\mathrm{AMB}$ & Broadcast & $\begin{array}{l}\text { Saves cost as } \\
\text { repeaters are } \\
\text { not used at } \\
\text { intersections }\end{array}$ & $\begin{array}{l}\text { Wastage of } \\
\text { time in } \\
\text { finding the } \\
\text { vehicle } \\
\text { closest to the } \\
\text { intersection. }\end{array}$ & Yes & Yes \\
\hline UMB & Broadcast & $\begin{array}{l}\text { Saves } \\
\text { bandwidth } \\
\text { and reduce } \\
\text { redundancy } \\
\text { by } \\
\text { choosing one } \\
\text { vehicle for } \\
\text { forwarding } \\
\text { the } \\
\text { packet }\end{array}$ & $\begin{array}{l}\text { The cost } \\
\text { incurred on } \\
\text { installing } \\
\text { repeaters that } \\
\text { are used to } \\
\text { forward } \\
\text { packets at } \\
\text { intersections. }\end{array}$ & Yes & Yes \\
\hline ABSM & Broadcast & $\begin{array}{l}\text { It resolves } \\
\text { propagation } \\
\text { at the } \\
\text { intersection } \\
\text { without } \\
\text { recognizing } \\
\text { intersections. }\end{array}$ & $\begin{array}{l}\text { Protocol } \\
\text { overhead is } \\
\text { high when } \\
\text { there are } \\
\text { multiple } \\
\text { simultaneous } \\
\text { broadcasting } \\
\text { tasks. }\end{array}$ & Yes & Yes \\
\hline BEAM & Multicast & $\begin{array}{l}\text { Bandwidth is } \\
\text { utilized } \\
\text { efficiently } \\
\text { during an } \\
\text { emergency } \\
\text { situation and } \\
\text { vehicles } \\
\text { decide } \\
\text { themselves } \\
\text { whether to } \\
\text { receive the } \\
\text { message or } \\
\text { not. }\end{array}$ & $\begin{array}{l}\text { Maintenance } \\
\text { of } \\
\text { Multicast } \\
\text { group is } \\
\text { difficult. }\end{array}$ & Yes & Yes \\
\hline UGAD & Geocast & $\begin{array}{l}\text { Reduce } \\
\text { redundant } \\
\text { rebroadcast } \\
\text { and } \\
\text { increases } \\
\text { packet } \\
\text { arrival ratio }\end{array}$ & $\begin{array}{l}\text { There is no } \\
\text { method for } \\
\text { assuring that } \\
\text { message has } \\
\text { been reached } \\
\text { to destination } \\
\text { or not. }\end{array}$ & Yes & Yes \\
\hline
\end{tabular}

\section{Conclusion}

In this paper, we've discussed the challenge in data dissemination in vehicular network, existing mechanism of data dissemination and planned approach for information dissemination for main road eventualities for vehicular networks. We have margin unit, and cluster formations the proposed approach within the planned design V2I and V2V communications was done in line with data priority. We've got additionally planned the 
design for information dissemination in VANETs. The most benefit of mistreatment roadside unit (RSU) in so many approaches is to attain low latency communications among vehicle and extend their property. It is additionally helpful for distribution time important information. In this paper discuss a different type of data dissemination protocol. The data dissemination approaches are structure as Multicast, Geocast/Broadcast, and Unicast method. These protocols expose that completely different scattering procedures are utilized in different situations i.e. some protocol is useful for propagation information in the urban area whereas some are useful for the highway. Every protocol has its specific advantage as well as disadvantage. The future plan is seeking out the issues of any of those protocols and checks out to deal with the find out the problem.

\section{References}

[1] M. Chaqfeh, A. Lakas, A novel approach for scalable multi-hop data dissemination in vehicular ad hoc networks, Ad Hoc Networks (2015), pp.1570-8705.

[2] Pratibha Tomar, Brijesh Kumar Chaurasia and G. S. Tomar, "State of the Art of DataDissemination in VANETs", International Journal of Computer Theory and Engineering, Vol.2, No.6, December (2010),pp.1793-8201.

[3] Brij Bihari Dubey, Naveen Chauhan and Prashant Kumar, "A Survey on Data Dissemination Techniques used in VANETs" International Journal of Computer Applications, Vol.10, No.7, November (2010), pp. 321-326.

[4] Navneet Kaur, Amarpreet Singh, "A Survey on Data Dissemination Protocols used in VANETs", International Journal of Computer Applications, Volume 120, No.23, June (2015), pp. 43-50.

[5] Mor Annu, "Study of Different Type of Data Dissemination Strategy in VANET," International Journal of Engineering Science and Innovative Technology (IJEST), Vol.1, No.2, November Vol. 1, issue 2 (2012), pp. 6-8.

[6] Rakesh Kumar and Mayank Dave, "A Review of Various VANET Data Dissemination Protocols" International Journal of u- and e- Service, Science and Technology, Vol. 5, No. 3, September (2012), pp. 320-326.

[7] Wai Chen, Ratul K. Guha, Taek Jin Kwon, John Lee and Irene Y. Hsu,” A Survey and Challenges in Routing and Data Dissemination in Vehicular Ad-hoc Networks" IEEE International Conference on Vehicular Electronics and Safety Columbus, OH, USA. September 22-24, (2008), pp. 328-333.

[8] Yousef-Awwad, Daraghmi and Chih-Wei Yi," Forwarding Methods in Data Dissemination and Routing Protocols for Vehicular Ad Hoc Networks" IEEE Network, Vol. 27, no. 6, (2013), pp. 74-79.

[9] S. Panichpapiboon and W. Pattara-atikom, "A Review of Information Dissemination Protocols for Vehicular Ad Hoc Networks", IEEE Communications Surveys \& Tutorials, (2011), pp. 1-15.

[10] G. Korkmaz, E. Ekici and F. Ozg Uner, "An Efficient Fully Ad-hoc Multi-Hop Broadcast Protocol for Inter-Vehicular Comm. Sys.", Proc. of IEEE International Conf. on Comm. (ICC), (2006), pp. 423-428.

[11] Q. Yu and G. Heijenk, "Abiding Geocast for Warning Message Dissemination in Vehicular Ad Hoc Networks", Proc. of IEEE Int'l Conf. on Comm. (ICC), (2008), pp. 400-404.

[12] D. Li, H. Huang, X. Li, M. Li and F. Tang, "A Distance-Based Directional Broadcast Protocol for Urban Vehicular Ad Hoc Network", Proc. of IEEE International Conf. on Wireless Communication, Networking and Mobile Computing (WiCom), (2007), pp. 1520-1523.

[13] M. Saito, J. Tsukamoto, T. Umedu, and T. Higashiro, "Evaluation of dissemination based inter-vehicle ad-hoc communication protocol for local traffic information services", Proceedings of IEEE AINA, (2010), pp. 76-87.

[14] Ryosuke Akamatsu, Masaki Suzuki, Takuya Okamoto, Koichiro Hara, Hiroshi Shigeno, "Adaptive Delay- based Geocast Protocol for Data Dissemination in Urban VANET", Proc. of IEEE Seventh International Conference on Mobile Computing and Ubiquitous Networking (ICMU), (2014), pp.141146. 


\section{Authors}

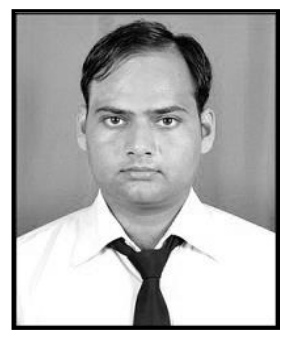

Hari Krishna, is currently pursuing M. TECH in Electronics and Communication Engineering with Spl. in Wireless Communication Systems at Lovely Professional University, India. His research interests include Ad-hoc Networks and Cryptography

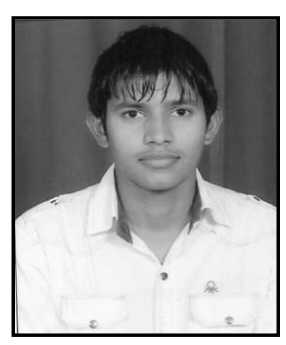

Kuldeep Kumar, is currently pursuing M. TECH in Electronics and Communication Engineering with Spl. in Wireless Communication Systems at Lovely Professional University, India. His research interests include Adhoc Networks and Cryptography.

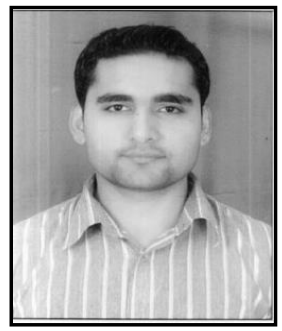

Sandeep Kumar Arora, is currently pursuing $\mathrm{Ph}$. D. in Electronics \& Electrical Engineering with Spl. in Design of Secure Initiation Protocol in VANET. He is working as an Asst. Prof. in Lovely Professional University since 2011. His research interest includes Wireless Sensor Networks, Computer Networks, Adhoc Networks Communications and Cryptography. He is an author of more than one dozen research papers indexed in Scopus. 